Reprad. Nutr. Dévelop., 1980, 20 (6), 1883-1892.

\title{
Estimation de la production laitière de la jument allaitante par marquage de l'eau corporelle du poulain
}

\author{
par M. DOREAU, G. DUSSAP **
}

arec la collaboration technique de H. DUBROEUCQ *

Laborafoire de la Production laitière, I.N.R.A.,

* Laboratoire de la Production de Viande, I.N.R.A., Theix, Saint-Genès-Champanelle, 63110 Beaumont, France

** Laboratoire de Génie chimique ef biologique, Université Clermont II, Complexe Scientifique des Cézeaux, BP 45, 63170 Aubière, France.

Summary. Estimation of nursing mare milk production using deuterium oxide as a tracer of offspring body water.

Fermale milk production was related to offspring water turnover, estimated by a tracer method (fig. 1). After deuterium oxide was injected into the blood of the offspring, we measured the decrease of its concentration in time. Milk intake was calculated by equations taking into account the weight gain of the offspring.

The reliability of this method was checked in 28 bottle-fed lambs with known milk intake; the correlation between the actual intakes and our estimates was 0.98 with an error means of 5.6 p. 100. The error on the mean of 28 lambs was 0.5 p. 100 (table 1). The milk production of 6 heavy brood mares, estimated during the first week of lactation, varied from 12 to $20 \mathrm{~kg}$ per day between animals (table 2).

This method did not interfere with the mother-offspring relationship nor with the usual suckling conditions ; it can be used advantageously during the onset of lactation in species whose offspring suck frequently.

\section{Introduction.}

La connaissance de la production laitière de la jument est essentielle d'une part pour estimer ses besoins en lactation, jusqu'à présent calculés d'après ceux de la vache (N. R. C. 1973), d'autre parf pour expliquer la vitesse de croissance élevée du du poulain sous la mère et ses variations : de $2000 \mathrm{~g} / \mathrm{j}$ à la naissance à $1000 \mathrm{~g} / \mathrm{j}$ au sevrage (Martin-Rosset, Doreau ef Cloix, 1978).

La production laitière estimée par différence de poids du poulain avant et après tétées est difficile à obtenir avec précision en début de lactation. En effet, le poulain tète plusieurs fois par heure pendant le premier mois (Martin-Rosset, Doreau et Cloix, 1978) et la mamelle, de faible capacité - environ 21 . — se remplit en moins de $2 \mathrm{~h}$ (Ala- 
guzhin, 1964 ; Storch, 1969) ; or, les mesures ont été réalisées au mieux à intervalles de 2 à 3 h (Flade, 1955 ; Tcherepanova, 1961). Pour les mêmes raisons, la traite fournit une mesure imprécise ; en outre, elle présente l'inconvénient de nécessiter une adaptation de l'animal (Masloboev, 1961) et la relation avec la quantité de lait bue par le poulain est très incertaine. Lorsque la traite est précédée d'une injection d'ocytocine, le lait recueilli comporte une fraction de lait résiduel qui n'aurait pas été bu par le jeune : 6 à 10 p. 100 chez le veau (Le Neindre, 1973). L'estimation de la production laitière d'après la croissance du poulain, par transposition des résultats obtenus sur bovins (Leonhard, 1956) peut conduire à des résultałs très erronés. Une relation entre la quantité de lait bue par le poulain ef la durée journalière de tétée semble également difficile à établir : la viłesse d'ingestion de lait est très variable avec l'âge ef la race des poulains (Rogalski, 1973), ef probablement avec les individus. La méthode de double marquage du laif utilisée par Nicol et Irvine (1973) permet la mesure de quantités de lait bues lors d'une tétée et présente donc des inconvénients similaires à la méthode de pesées avant et après tétées.

Ces considérations nous ont amenés à choisir une méthode qui respecte l'allaitement naturel permanent, risquant peu a priori de modifier le comportement du couple mère-jeune, ef de mise en cuvre simple. Elle consiste à injecter au poulain un marqueur de l'eau et à estimer la quantité de lait bue à partir de la vitesse de renouvellement de l'eau corporelle. Macfarlane, Howard et Siebert (1969) sur des ovins puis Holleman, White ef Luick (1976) sur des bovins ont décrit une felle méthode, fondée sur la décroissance exponentielle de la concentration du marqueur dans l'organisme. Nous avons appliqué au poulain un mode de calcul voisin en tenant compte de son gain de poids. A partir d'un certain nombre d'hypothèses, nous avons représenté l'évolution du volume d'eau corporelle par un modèle à compartiment. II nous a alors été possible d'estimer la quantité de lait bue par le poulain sous la mère, et par conséquent la production laitière de la jument, à partir de la vitesse d'élimination du marqueur de l'organisme du jeune, et de certains autres paramètres : quantité de marqueur injectée, poids du poulain, teneur en matière sèche et composition du lait.

A partir de cette méthode nous avons vérifié sur, 13 agneaux nourris au biberon, la validité de la relation mathématique que nous avons établie puis estimé la quantité de lait bue par 6 poulains sous la mère.

\section{Principe de la méthode.}

Utilisation d'un marqueur de l'eau corporelle. - Parmi les différents marqueurs de l'eau utilisés jusqu'à présent, seules les eaux isotopiques (deutériée ou lourde, et tritiée) présentent un espace de diffusion voisin de celui de l'eau quoique légèrement supérieur (Panaretto et Till, 1963 ; Haxhe, 1964). Ils s'homogénéisent en quelques heures à l'eau de tous les compartiments hydriques de l'organisme, dont le sang ef les produits d'excrétion (Robelin, 1973). Ces propriétés sont identiques pour l'eau lourde et l'eau tritiée. Contrairement à la majorité des expérimentateurs, nous avons choisi l'eau lourde, qui présente l'avantage de ne pas être radioactive ; sa détermination est en revanche plus longue et délicate que celle de l'eau tritiée. 
Modèle à compartiment des mouvements de l'eau dans l'organisme (fig. 1, 2). - La méthode utilisée consiste à décrire les entrées ef sorties d'eau au niveau du compartiment hydrique corporel du jeune, de taille variable puisque l'animal est en croissance. Afin de pouvoir utiliser un modèle mathématique simple, on doit admettre que l'apport d'eau par le lait et l'excrétion d'eau (urine, fèces, sueur, vapeur d'eau) sont des phénomènes continus. Ces hypothèses peuvent être acceptées pour une période expérimentale suffisamment longue ( 4 à 6 jours dans notre cas) : en effet, les tétées s'effectuent en début de lactation à une fréquence très élevée ; les modes d'excrétion sont diversifiés et certains d'entre eux s'effectuent en permanence (sueur, vapeur d'eau). Des fluctuations nycthémérales existent cependont (Theriez, non publié). Afin d'en éliminer l'effet, il suffit de mesurer la concentraicicn en marqueur chaque jour à la même heure.

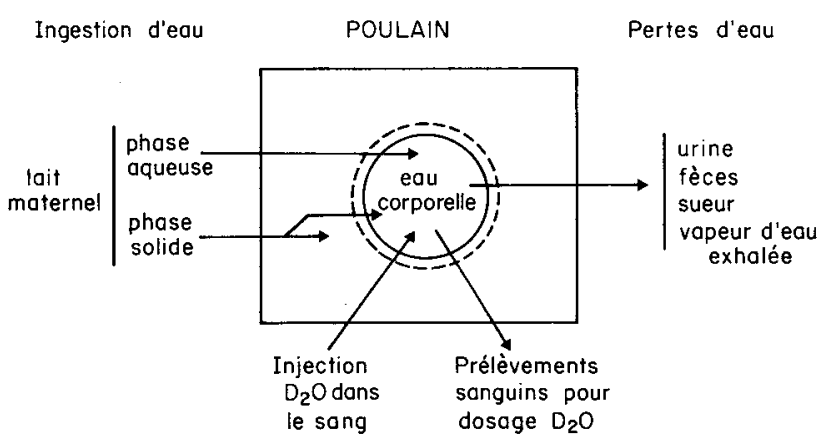

FIG. 1. - Mesure de la production laitière de la jument à partir de l'estimation des quantités de lait bues par le poulain. Principe de la méthode.

Afin de simplifier la formulation, on considère la proportion d'eau dans l'organisme du jeune comme constante tout au long de la mesure. Les résultats de Meyer et Ahlswede (1976) sur la diminution de la teneur en eau du poulain durant le mois précédant la naissance $(1,1$ point $p .100)$ permettent de penser que cette hypothèse n'entraînera qu'une erreur minime. Il est alors possible d'écrire :

$$
\frac{V_{0}}{P_{o}}=\frac{V_{t}}{P_{t}}
$$

$V$ et $P$ correspondant respectivement au volume d'eau corporelle et au poids vif du poulain, mesurés aux temps 0 et $t$.

Formules d'estimation de la quantité d'eau ingérée. - Chez le jeune animal, on peut considérer que, sur une courte période de mesure, les quantités d'eau ingérée $I$ et excrétée $E$, et par conséquent les variations de poids, augmentent de manière linéaire. Ceci se traduit par :

$$
\mathrm{dl}=\mathrm{k}_{\mathrm{i}} \mathrm{dt} \quad \mathrm{dE}=\mathrm{k}_{\mathrm{e}} \mathrm{dt}
$$

$k_{i}$ ef $k_{e}$ sont les constantes de vitesse d'ingestion et d'excrétion d'eau corporelle.

Les mouvements d'eau peuvent être définis par un bilan de l'eau totale et un bilan du marqueur. 


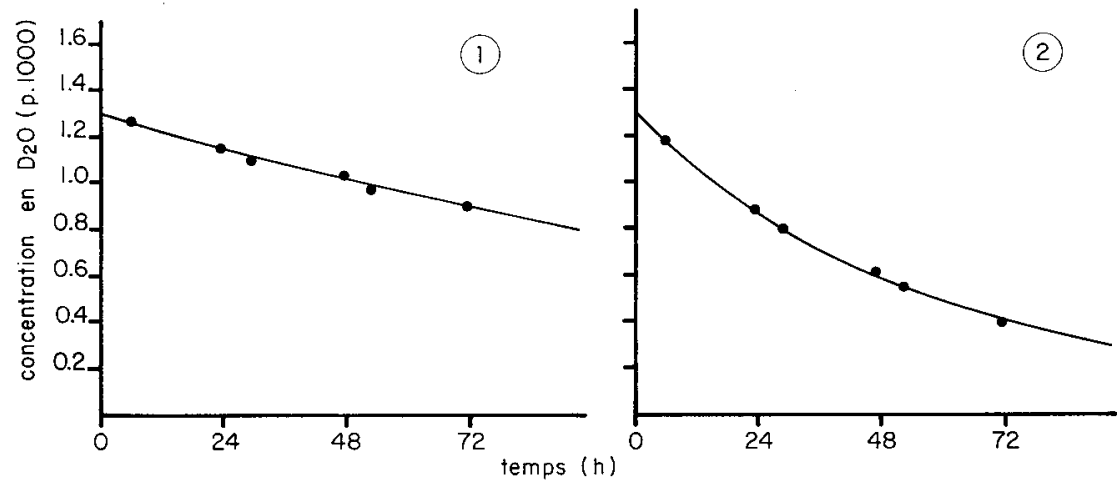

FIG. 2. - Deux exemples d'ajustement des concentrations en sau lourde à la courbe théorique.

1) Agneau de $4,6 \mathrm{~kg}$ consommant $425 \mathrm{~g}$ d'eau par jour.

'2) Agneau de 7,2 kg consommant $2196 \mathrm{~g}$ d'eau par jour.

Le bilan de l'eau s'écrit :

$$
\frac{d V}{d t}=k_{i}-k_{e} \text { soit } V=V_{o}+\left(k_{i}-k_{e}\right) \dagger
$$

Le bilan du marqueur s'écrit :

$$
\frac{d(V C)}{d t}=-k_{e} C=\frac{C d V}{d t}+\frac{V d C}{d t}
$$

$C$ étant la concentration en marqueur au temps t.

Dans le cas - rare - où l'animal garde un poids constant pendant la mesure, $\mathrm{k}_{\mathrm{i}}=\mathrm{k}_{\mathrm{e}}$ et $\mathrm{V}=\mathrm{V}_{\mathrm{o}}$ :

$$
\frac{v_{0} d C}{d t}=-k_{e} C \text {. }
$$

Ceci conduit à

$$
C=\lambda_{1} \mathrm{e}^{\frac{-\mathbf{k}_{\mathrm{i}} t}{\mathrm{~V}_{\mathrm{o}}}}
$$

pour $t=0, \lambda_{1}=C_{0}$.

On obtient :

$$
\mathrm{C}=\mathrm{C}_{0} \mathrm{e}^{\frac{-\mathrm{k}_{\mathrm{i}} \mathrm{t}}{\mathrm{V}_{\mathrm{o}}}} \text { ou } \mathrm{C}=\mathrm{C}_{0} \mathrm{e}^{\frac{-\mathrm{k}_{\mathrm{i}} \mathrm{C}_{0} \mathrm{t}}{\mathrm{Q}_{0}}}
$$

$Q_{0}$ ef $C_{0}$ étant la quantité de marqueur injectée et sa concentration initiale.

Dans le cas général où le poids de l'animal varie :

$$
V=V_{0}+\left(k_{i}-k_{e}\right) \dagger \text {. }
$$

Comme

$$
\begin{gathered}
\frac{d V}{d t}=k_{i}-k_{e}, \frac{V d C}{d t}=-k_{i} C \text { et } \frac{d C}{d t}=\frac{-k_{i} C}{V_{o}+\left(k_{i}-k_{e}\right) t} \\
\frac{d C}{d t}=\frac{-k_{i} d t}{V_{o}+\left(k_{i}-k_{e}\right) t} \text { ef } \log C=\frac{-k_{i}}{k_{i}-k_{e}} \log \left[\left(k_{i}-k_{e}\right) t+V_{0}\right]+\lambda_{2} .
\end{gathered}
$$


Pour $\uparrow=0$,

$$
\lambda_{2}=\log C_{o}+\frac{k_{i}}{k_{i}-k_{e}} \log V_{o}
$$

Donc

et

$$
\log \frac{C}{C_{0}}=\log \left[\frac{\left(k_{i}-k_{e}\right) t+V_{0}}{V_{o}}\right]^{\frac{-k_{i}}{k_{i}-k_{e}}}
$$

$$
\begin{gathered}
C=C_{0}\left[\frac{\left(k_{i}-k_{e}\right) t+V_{0}}{V_{0}}\right]^{\frac{-k_{i}}{k_{i}-k_{e}}} \\
C=C_{0}\left[\frac{\left(k_{i}-k_{e}\right) t+\frac{Q_{0}}{C_{0}}}{\frac{Q_{0}}{C_{o}}}\right]^{\frac{-k_{i}}{k_{i}-k_{e}}} .
\end{gathered}
$$

Par ailleurs, entre les temps 0 ef $T$ (début et fin de la mesure)

soit :

$$
\left(k_{i}-k_{e}\right) T=V_{T}-V_{o}=V_{0}\left(\frac{P_{T}}{P_{o}}-1\right) \text {. }
$$

$$
\left(k_{i}-k_{e}\right) T=\frac{Q_{0}}{C_{0}}\left(\frac{P_{T}}{P_{0}}-1\right) \text {. }
$$

En remplaçant dans la relation (2) $k_{i}-k_{e}$ par sa valeur exprimée dans la relation (3), il esł possible de connaître $k_{i}$ à partir de $Q_{0}, P_{0}, P_{T}, T$ et des concentrations $C$ à différents temps $t$; lorsque $P_{0}=P_{T}$ on utilise la relation (1).

Relation entre l'apport d'eau à l'organisme ef la consommation de lait. - L'eau provenant du lait ingéré a une double origine : d'une part, la fraction aqueuse du lait, d'autre part, l'eau produite par le métabolisme des nutriments du lait. Le catabolisme oxydatif produit de l'eau à raison respectivement de 58,107 ef $41 \mathrm{~g}$ d'eau pour $100 \mathrm{~g}$ de lacłose, matières grasses et matières azotées (Jacquot, Le Bars et Simonnet, 1958). Mais une partie seulement du lait est catabolisée, l'autre étant utilisée à la synthèse des protéines et lipides corporels.

L'apport énergétique des différents nutriments du lait est calculé à partir de sa teneur en lactose, matières grasses et matières azotées, qui apportent respectivement 3,$9 ; 9,5$ ef 5,7 kcal d'énergie brute par $\mathrm{g}$ (Vermorel et Patureau-Mirand, 1978). Le devenir de cette énergie dépend des proportions de son utilisation pour l'entretien et la croissance, du rendement d'utilisation de l'énergie métabolisable et la proportion d'énergie fixée sous forme de lipides ou de protéines. Ainsi, il est possible de chiffrer pour chaque nutriment la proportion fixée et catabolisée avec formation d'eau. Le résultat dépend de l'espèce animale, de l'âge du jeune et de son gain de poids journalier.

Connaissant la teneur en matière sèche $(S)$ du lait et ayant estimé la proportion (M) d'eau d'origine métabolique (en $\mathrm{g}$ p. $100 \mathrm{~g}$ de lait) il est possible de déterminer la quantité journalière $(L)$ de lait bue par le jeune :

$$
L=\left(\frac{100}{100-S+M}\right) k_{i} \text {. }
$$




\section{Schéma expérimental.}

Vérification de la méthode sur agneaux. - L'utilisation d'agneaux pour étalonner la méthode est justifiée dans la mesure où la proportion d'eau varie lentement chez l'agneau (1 point p. 100 entre 1 et 5 semaines : Robelin, 1977) et où les tétées sont relativement nombreuses (environ 20/j : Ricordeau, Boccard et Denamur, 1960). II esł donc probable que, si la méthode est fiable pour le couple brebis-agneau, elle le sera pour le couple jument-poulain.

Nous avons utilisé les résultats d'un essai de Theriez ef al. (non publié) sur agneaux âgés de 3 semaines et recevant ad libitum un alimient d'allaitement à 10,16 ou 22 p. 100 de matière sèche (afin d'obtenir une plage importante de quantité d'eau bue) distribué au biberon. Les quantités ingérées ont été mesurées. 28 agneaux ont consommé leur aliment d'allaitement de manière régulière. Après injection d'eau lourde à raison de 0,6 p. 1000 environ du poids vif, 6 prélèvements de sang suivis de pensées des agneaux se sont échelonnés sur $72 \mathrm{~h}$. La quantité d'eau lourde injectée a été mesurée exactement sur 15 agneaux seulement. Sur les 13 autres, afin d'appliquer notre relation, nous avons dô estimer le volume hydrique corporel pour chaque agneau, en utilisant une équation de régression établie sur agneaux de même âge et même race.

L'eau du sang entier a été extraite par lyophilisation (Tissier ef al., 1978a) ef dosée par spectrophotométrie dans l'infrarouge (Thornton et Condon, 1950) sur appareil Perkin Elmer 180, selon la technique automatisée de Tissier et al. (1978a).

L'estimation de la production d'eau métabolique a été effectuée en considérant que les parts d'utilisation de l'énergie pour l'entretien et la croissance sont respectivement de 44 ef 56 p. 100 (d'après Tissier ef al., 1978b), que 70 p. 100 de l'énergie utilisée pour la croissance était fixée et que les parts de protéines et de lipides fixés étaient respectivement de 60 ef 40 p. 100 (Walker et Jagusch, 1969)

Estimation de la production laitière chez la jument. - A six poulains de mère Comtoise Bretonne ou Ardennaise, maintenus en box avec leur mère ef âgés de 36 à 60 h, nous avons injecté de l'eau lourde stérile apyrogène, selon la technique utilisée pour les agneaux. Trois des mesures ont été réalisées en 1977, trois en 1978 . Sur les 3 poulains ułilisés en 1977, nous avons effectué 8 prélèvements de $10 \mathrm{ml}$ de sang situés $2,4,6,8$, $24,48,72$ et $96 \mathrm{~h}$ après l'injection. Les quatre premiers étaient destinés à estimer le nombre d'heures nécessaires pour obtenir une diffusion complète dans l'organisme, les quatre suivants à ajuster au mieux les coefficients de l'équation de décroissance de la concentration en marqueur et à dépister d'éventuelles valeurs aberrantes. Avant l'injection et lors des quatre dernières prises de sang, nous avons pesé les poulains. Deux échantillons de lait de la jument ont été prélevés par traite, en début et en fin de mesure. L'état de santé ef l'absence de modifications du comportement ont été contrôlés tous les jours (température rectale, rythme respiratoire et cardiaque). Aucune perturbation des poulains n'a été mise en évidence. Sur les 3 animaux utilisés en 1978, les prises de sang ont eu lieu $2 \mathrm{~h}$ après l'injection, puis à intervalles de 24 à $48 \mathrm{~h}$ pendant 4 à 6 jours. Les pesées ont été effectuées à l'injection puis au moment des prises de sang les jours suivants. Les échantillons de lait ont été prélevés comme en 1977. 
L'eau lourde a été dosée par la méthode exposée précédemment. Sur chaque échantillon de lait, la teneur en matière sèche a été mesurée, mais pas la teneur en éléments nutritifs. Comme nous ne disposions pas d'informations sur la représentativité de l'échantillon prélevé, nous avons préféré estimer les proportions relatives en lactose, matières grasses et protéines d'après les valeurs moyennes de la bibliographie (Ullrey et al., 1966) : respectivement 6,$0 ; 1,9$ et 2,6 p. 100. L'énergie a donc été ingérée sous forme de 42 p. 100 de lactose, 31 p. 100 de matières grasses ef 27 p. 100 de protéines. Nous avons considéré que les parts d'utilisation de l'énergie pour l'entretien et la croissance étaient de 28 et 72 p. 100 (N.R.C., 1973).

En l'absence de données cohérentes relatives au poulain, nous avons admis que, comme chez le jeune ruminant, 70 p. 100 de l'énergie utilisée pour la croissance était fixée et que, à la naissance, les parts de protéines et de lipides fixés étaient de 60 et 40 p. 100. Du mode de calcul exposé précédemment on déduit que la totalité du lactose et $2 / 3$ des matières grasses sont oxydés dans l'organisme du poulain. La production d'eau métabolique s'élève alors à $46 \mathrm{~g}$ par $\mathrm{kg}$ de lait.

\section{Résultats.}

Vérification de la méthode sur agneaux (tabl. 1). - Les quantités de lait bues ont été estimées à partir de la méthode classique employée par Macfarlane, Howard et Siebert (1969) (relation I) et de la méthode présentée dans ce texte (relations II).

La précision de l'ajustement des concentrations en eau lourde à l'équation théorique que nous avons exposée a été plus ou moins grande selon les animaux : le coefficient de corrélation $r^{2}$ a été supérieur à 0,98 dans 22 cas sur 28 . L'ajustement est meilleur dans la majorité des cas que celui obtenu par la relation 1.

TABLEAU 1

Estimation sur 28 agneaux des quantités de lait bues.

Comparaison de deux méthodes de marquage de l'eau corporelle du jeune

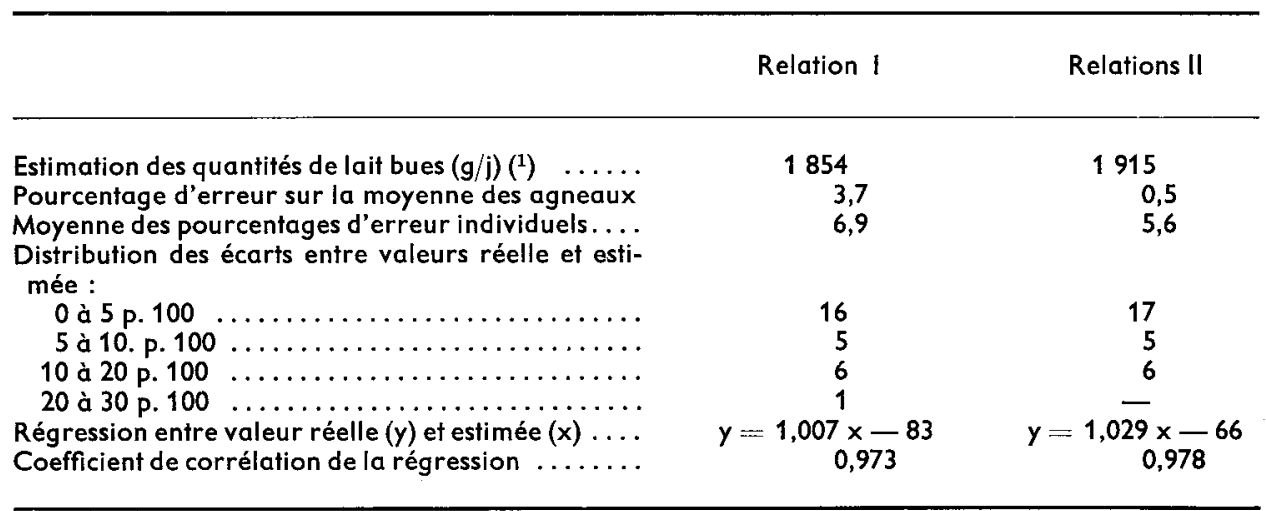

(1) Quantité de lait réellement bue : $1925 \mathrm{~g} / \mathrm{j}$.

Relation I : l'estimation ne tient pas compte des variations de poids de l'agneau (Macfarlane, Howard ef Siebert, 1969). Relations II : I'estimation tient compte des variations de poids de l'agneau (cf. texte). 
II semble que les relations II permettent une meilleure prévision des quantités ingérées que la relation I, l'erreur sur la valeur moyenne n'étant que de 0,5 p. 100. Il est à noter cependant que la pente de la régression est plus proche de 1 avec la relation 1. La précision des estimations individuelles ne met en évidence qu'une faible différence entre les deux méthodes. La distribution des écarts à la moyenne montre un nombre assez élevé d'erreurs de plus de 10 p. 100 par rapport aux quantités ingérées. Il peut en partie être attribué à l'estimation approximative du volume hydrique corporel à partir du poids vif sur 13 animaux, obtenue, selon Robelin (1977), avec un coefficient de variation résiduel de 3 p. 100.

Estimation de la production laitière chez la jument (tabl. 2). - Les valeurs expérimentales des concentrations en eau lourde s'ajustent correctement à la courbe théorique : pour un même animal, sur 4 mesures espacées de 24 h, le coefficient de corrélation est toujours supérieur à 0,99 . Le coefficient $k_{i}$ calculé entre deux prises de sang espacées de $24 \mathrm{~h}$ varie de moins de $1 \mathrm{p}$. 100 d'un jour à l'autre. Les points 2, 4, 6 et $8 \mathrm{~h}$ se situent sur la courbe théorique; la diffusion du marqueur dans l'eau corporelle semble donc complète en moins de $2 \mathrm{~h}$. La durée de demi-vie biologique du marqueur, proche de $60 \mathrm{~h}$ en moyenne, varie entre 45 et $75 \mathrm{~h}$ selon les animaux. L'élimination complète du marqueur est longue : en moyenne plus de 12 jours sont nécessaires pour obtenir la disparition de 95 p. 100 de marqueur.

TABLEAU 2

Quantité de lait bue, gain de poids ef proportion d'eau corporelle des poulains, élimination du marqueur

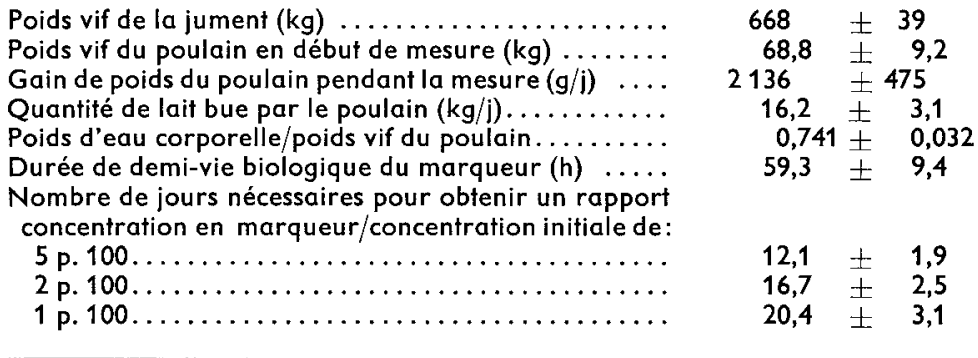

Les quantités de lait bues en première semaine par les poulains, en moyenne de $16,2 \mathrm{~kg}$, varient entre $12 \mathrm{et} 20 \mathrm{~kg} / \mathrm{j}$. La proportion d'eau corporelle dans l'organisme du poulain, voisine de 74 p. 100, varie fortement avec les individus : 71 à 79 p. 100. Pendant la période de mesures, le gain de poids journalier des poulains se situe entre 1600 et $2900 \mathrm{~g} / \mathrm{j}$. La liaison entre quantité de lait bue et croissance du poulain en première semaine, définie par le coefficient de corrélation des rangs $\left(r_{\mathrm{s}}=0,77\right.$; $n=6$ ), n'est pas significative au seuil de 5 p. 100 .

\section{Discussion.}

Le marquage de l'eau corporelle du jeune semble être une méthode intéressante d'estimation de la quantité de lait bue et donc de la production laitière de la mère. 
En effet, la relation obtenue sur les agneaux entre les quantités réellement bues et leur estimation est étroite, comme celle obtenue par Macfarlane, Howard et Siebert (1969) : $r=0,96$. Les consommations de lait peuvent être obtenues avec une erreur faible et sans biais important, bien que nous n'ayons pas tenu comple de la légère surestimation du volume hydrique corporel par l'espace de diffusion de l'eau lourde. Cette méthode a par ailleurs permis de quantifier l'erreur due à l'estimation ; la méthode de pesées avant et après tétée ne permet de la quantifier qu'au niveau d'une tétée (Moss, 1977).

Parmi les sources d'imprécision de la méthode de marquage de l'eau corporelle on peut citer :

- les variations au cours de la journée des quantités d'eau bue et excrétée, d'où peut résulter un médiocre ajustement des concentrations en eau lourde à la courbe théorique. On peut penser que les meilleurs ajustements obtenus avec les poulains par rapport aux agneaux sont dus à une répartition plus régulière de l'ingestion dans le cas de l'allaitement naturel que de l'allaitement artificiel ;

- la connaissance de la teneur en matière sèche et de la composition du lait maternel, de la production d'eau par les différents nutriments (Van Es, 1969) et de la part des nutriments oxydée et fixée par le jeune. Les variations sont importantes en première semaine de la lactation ; elles peuvent en partie expliquer la faible liaison entre les quantités de lait bues et la croissance des poulains ;

- l'incertitude sur le dosage de l'eau lourde, bien que, selon Tissier ef al. (1978a), sa précision soit de l'ordre de 1 p. 100, ainsi que sur les pesées des jeunes, quoiqu'elle influe peu sur la valeur de $k_{i}$. Il semble cependant que cette méthode soit la plus fiable, même si l'on utilise la relation plus simple employée par Macfarlane, Howard ef Siebert (1969). Néanmoins, elle n'est applicable que lorsque le lait est la seule source d'eau pour le jeune ou que les autres entrées d'eau dans l'animal sont mesurées ; ceci ne peut être obtenu qu'arec des animaux en stabulation. Dans le cas d'animaux au pâturage, des méthodes de double marquage - un marqueur injecté à la mère ef l'autre au jeune — doivent être utilisées (Holleman, White et Luick, 1975 ; Wright et Wolff, 1976).

Reçu en février 1980

Accepté en juillet 1980.

Note : Une méthode similaire a récemment été développée par Dove et Freer (1979).

Remerciements. - Nous tenons à remercier J. P. Brun, Y. Houssin ef $M$. Theriez (Laboratoire de la Production Ovine, INRA, Theix) qui ont mis à notre disposition les résultats expérimentaux relatifs aux agneaux ef J. Kopp (Station de Recherches sur la Viande, INRA, Theix) qui a réalisé le programme d'ajustement de courbes que nous avons utilisé.

\section{Références}

ALAGUZHIN A., 1964. Production laitière des jument Lokaï (en russe). Konevodsivo, 34, $13-14$.

DOVE M., FREER M., 1979. The accuracy of fritiated water turnover rate as an estimate of milk intake in lambs. Austr. J. agric. Res., 30, 725-739.

FLADE E., 1955. Milchleistung und Milchqualität bei Stuten. Tierzucht, 9, 381-383.

HAXHE J. J., 1964. Mesures des compartiments corporels. Méthodes et résultats. J. Physiol. Paris, 56, 7-109. 
HOLLEMAN D. F., WHITE R. G., LUICK J. R., 1975. New isotope methods for estimating milk intake and yield. J. Dairy Sci., 58, 1814-1821.

JACQUOT R., LE BARS H., SIMONNET H., 1958. Nutrition animale, Vol. II, Tome 1. Ed. Baillière, Paris.

LE NEINDRE P., 1973. Observations sur l'estimation de la production laitière des vaches allaitantes par la pesée du veau avant et après la tétée. Ann. Zootech., 22, 413-422.

LEONHARD I., 1956. Etudes préliminaires de la production et de la composition chimique du lait de jument (en polonais). Roczn. Nauk. Roln., B, 70, 333-346.

MACFARLANE W. V., HOWARD B., SIEBERT B. D., 1969. Tritiafed water in the measurement of milk intake and tissue growth of ruminants in the field. Nature, 221, 578-579.

MARTIN-ROSSET W., DOREAU M., CLOIX J., 1978. Etude des activités d'un troupeau de poulinières de trait el de leurs poulains au pâturage. Ann. Żootech., 27, 33-45.

MASLOBOEV A. J., 1961. Caractéristiques de la lactation de la jument (en russe). Trudy Vses. Inst. Konevodsiva, 23, 135-150, In Nutr. Abstr. Rev., 1962, 32, 2775 (abstr.).

MEYER H., AHLSWEDE L., 1976. Über das intrauterine Wachstum und die Körperzusammensetzung von Fohlen sowie den Nährstoffbedarf tragender Stuten. Ubers. Tierernährg., 4, 263-292.

MOSS R. J., 1977. Rearing dairy replacements on the Atherton Tableland, Queensland. 2. Effect of number of calves per cow and grain supplementation. Austr. J. exp. Agric. anim. Husb., 17, 367-372.

NICOL A. M., IRVINE C. H. G., 1973. Measurement of the milk consumption of suckling beef calves by an isotope dilution method. Proc. N. Z. Soc. anim. Prod., 33, 176-183.

N. R. C., 1973. Nutrient requirements of horses. Ed. Nat. Acad. Sci., Washington.

PANARETTO B. A., TILL A. R., 1963. Body composition in vivo : II. The composition of mature goats and its relationship to the antipyrine, tritiated water and $\mathrm{N}$-acetyl-4-aminoantipyrine spaces. Austr. J. agric. Res., 14, 926-943.

RICORDEAU G., BOCCARD R., DENAMUR R., 1960. Mesure de la production laitière des brebis pendant la période d'allaitement. Ann. Zootech., 9, 91-120.

ROBELIN J., 1973. Estimation de la composition corporelle des animaux à partir des espaces de diffusion de l'eau marquée. Ann. Biol. anim. Bioch. Biophys., 13, 285-305.

ROBELIN J., 1977. Estimation in vivo de la composition corporelle des agneaux à partir de l'espace de diffusion de l'eau lourde. Ann. Biol. anim. Bioch. Biophys., 17, 95-105.

ROGALSKI M., 1973. Comportement du poulain au pâturage (en polonais). Przegl. Hodow., 41, 14-15.

STORCH R., 1969. Über das maschinelle Melken von Stuten. Milchwissenschaft, 24, 145-146.

TCHEREPANOVA V., 1961. L'alimentation et la production laitière des juments (en russe), Konevodsivo, 6, 11.

THORNTON V., CONDON F. E., 1950. Infra-red spectrometric determination of deuterium oxide in water. Anal. Chem., 22, 690-691.

TISSIER M., ROBELIN J., PURROY A., GEAY Y., 1978a. Extraction et dosage automatique rapide de l'eau lourde dans les liquides biologiques. Ann. Biol. anim. Bioch. Biophys., 18, 1223-1228.

TISSIER M., THÉRIEZ M., GUÉGUEN L., MOLÉNAT G., 1978 b. Ovins. In INRA, Alimentation des Ruminants, 403-448, Ed. INRA Publications. Versailles.

ULLREY D. E., STRUTHERS R. D., HENDRICKS D. G., BRENT B. E., 1966. Composition of mare's milk J. anim. Sci., 25, 217-221.

VAN ES A. J. H., 1969. Constants and factors regarding metabolic water, 513-514, In BLAXTER K.L., KIELANOWSKI J., THORBEK G., Energy metabolism of farm animals. Ed. Oriel Press, Newcastle.

VERMOREL M., PATUREAU-MIRAND P., 1978. Utilisation des produits terminaux de la digestion des glucides et des lipides par le veau préruminant. In Le veau de boucherie, 63-73. Ed. INRA Publications, Versailles.

WALKER C. M., JAGUSCH K. T., 1969. Utilization of the metabolizable energy of cow's milk by the lamb, 187-193, In BLAXTER K. L., KIELANOWSKI J., THORBEK G., Energy metabolism of farm animals. Ed. Oriel Press, Newcastle.

WRIGHT D. E., WOLFF J. E., 1976. Measuring milk intake of lambs suckling grazing ewes by a double isotope method. Proc. N. Z. Soc. anim. Prod., 36, 99-102. 